\title{
Zuwachs an interventionell-radiologischer Expertise: 10. DeGIR-Zentrum zertifiziert
}

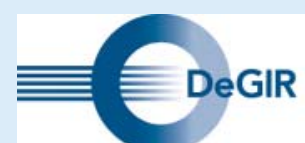

DEUTSCHE GESELLSCHAFT FÜR INTERVENTIONELLE RADIOLOGIE UND MINIMAL-INVASIVE THERAPIE

in der Deutschen Röntgengesellschaft e.V.

Es ist ein Gütesiegel für ausgewiesenes interventionell-radiologisches Wissen: das Zertifikat „DeGIR-Zentrum für interventionelle Gefäßmedizin und minimal-invasive Therapie“. Ausgezeichnet werden Kliniken, die interventionell-radiologische Behandlungen in der Gefäßmedizin, Schmerztherapie und bei onkologischen Erkrankungen in höchster Qualität anbieten. Seit Oktober 2017 darf sich nun auch das Institut für diagnostische und interventionelle Radiologie der DRK Kliniken Berlin/Köpenick mit dem Titel schmücken, und ist damit bereits das zehnte Zentrum in Deutschland. Die Zertifizierung weiterer Institute ist bereits in Planung.
Nach eingehender Prüfung vor Ort durch einen Auditor stellte die Deutsche Gesellschaft für Interventionelle Radiologie und minimal-invasive Therapie (DeGIR) das Zertifikat an das Institut für diagnostische und interventionelle Radiologie der DRK Kliniken Berlin/Köpenick aus. Institutsleiterin Dr. Kerstin Westphalen: „Ich würde Zertifizierungen unbedingt empfehlen. So können Radiologen auch nach außen ihre Qualifikation als kompetente Partner in der Patientenversorgung demonstrieren." Die Patienten können sich damit auf eine hohe Qualität in der interventionellen Behandlung verlassen. Denn Kliniken und radiologische Institute, die eine Zertifizierung anstreben, müssen strenge Kriterien erfül-

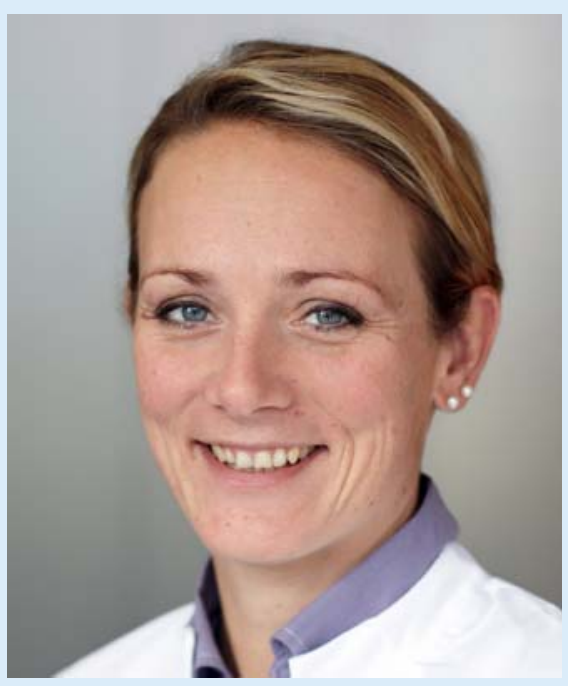

Dr. Kerstin Westphalen 
len. Dazu gehört die laufende qualitative Verbesserung, unter anderem durch den Nachweis von mindestens 250 Interventionen pro Jahr. Ergänzend müssen qualitätsrelevante Daten wie beispielsweise Komplikationen in der QualitätssicherungsDokumentation der DeGIR erfasst werden. Als erstes Zentrum wurde das Klinikum Saarbrücken gGmbH zertifiziert: „Besonders gut gefällt uns an der Zertifizierung als DeGIR-Zentrum die interdisziplinäre Ausrichtung des Gütesiegels und die Einbindung der übrigen Kliniken bei uns im Haus“, erklärt Prof. Dr. Elmar Spüntrup, Direktor des Instituts für Radiologie des Saarbrücker Klinikums. Jedes zertifizierte radiologische Institut verpflichtet sich, aktiv mit anderen klinischen Fachrichtungen zusammenzuarbeiten, beispielweise in Form interdisziplinärer Fallkonferenzen. Außerdem ist eine lückenlose Rund-umdie-Uhr-Betreuung erforderlich, in der alle Ressourcen für alle interventionellen Patienten zur Verfügung stehen.

\section{Laufende Fortbildungen sind Pflicht}

Um als DeGIR-Zentrum anerkannt zu werden, sind mindestens drei radiologische Fachärzte mit interventioneller Expertise vor Ort nötig. Ihr Fachwissen halten sie durch regelmäßige Fortbildungen auf dem neuesten Stand. Hierzu sind jährliche interne und externe Schulungen vorgeschrieben. Mindestens ein Radiologe muss eine Zertifizierung in mindestens einem der sechs DeGIR-Module der Stufe 2 nachweisen. In den Trainingsprogrammen für die Stufe 2 vertiefen interventionell spezialisierte Radiologen ihre Kenntnisse und Fertigkeiten in der bildgesteuerten minimalinvasiven Therapie. Das Zertifikat ist nach Ausstellung fünf Jahre gültig.

\section{Interventionsradiologische Fälle auf der Interaktiven Lehr- und Lernplattform}

Auch auf der Interaktiven Lehr- und Lernplattform der DRG sind didaktisch aufbereitete Fälle aus verschiedenen Bereichen der Interventions-Radiologie verfügbar. Anhand anschaulichen Bildmaterials werden die Teilnehmer Schritt für Schritt durch Indikationsstellung und Durchführung geleitet. In vielen Zwischenschritten werden je nach Fall Fragen z. B. zum jeweiligen Krankheitsbild, dem zu wählenden Verfahren, den einzelnen Interventionsschritten, zum Komplikationsmanagement sowie zum Outcome nach der jeweiligen Intervention gestellt. Die Teilnehmer erhalten sofort Feedback, ob sie die Fragen korrekt beantwortet haben. Am Ende erfolgt eine kurze Zusammenfassung inklusive Tipps zum Vertiefen des Gelernten. Die Zahl der Fälle wird ständig erweitert. Die interventions-radiologischen Fälle sind auf www.drg.de nach Mitglieder-Login kostenlos verfügbar.

\section{Interview}

\section{„Radiologen sollten endlich aus ihrem Schattendasein treten"}

Drei Fragen an... Dr. Kerstin Westphalen, Chefärztin des Instituts für diagnostische und interventionelle Radiologie am DRK Kliniken Berlin/Köpenick

Frau Dr. Westphalen, Ihr Institut trägt nun als zehnte Einrichtung die Auszeichnung „DeGIR-Zentrum“. Warum haben Sie sich für diese Zertifizierung entschieden?

Einerseits möchte ich die interventionelle Radiologie stärker publik machen. Radiologen sollten endlich aus ihrem Schattenda- sein treten und sich beispielsweise mit einer Zertifizierung als kompetente Partner in der Patientenversorgung präsentieren. Andererseits können mein Team und ich bei jeder Zertifizierung auch viel dazulernen. Ich habe während des Prozesses wertvolle Anregungen bekommen, beispielsweise zur klareren Definition von Arbeitsabläufen. Das erhöht natürlich die Qualität unserer radiologischen Leistungen. Wir haben uns außerdem parallel als Gefäßzentrum zertifizieren lassen, die Kombination spart Aufwand. Das Audit unserer Zertifizierung als „DeGIR-Zentrum“ fand in einer äußerst angenehmen und kollegialen Atmosphäre statt. Am meisten hat mich ein Kommentar des Auditors in seinem Bericht gefreut: „Die Chefärztin hat ihr Herz in der Interventionellen Radiologie, was sich positiv auf alle Bereiche auswirkt.“

Planen Sie nach der Zertifizierung, den Schwerpunkt interventionelle Radiologie auszubauen und versprechen Sie sich davon Vorteile, z. B. in der Patientengewinnung?

Mir ist es ein Anliegen, für das faszinierende Fachgebiet der Interventionellen Radiologie zu werben. Hoffentlich gelingt es mir, meine Begeisterung auch auf junge ärztliche Kollegen zu übertragen. Meine Klinik plant, nun auch eine DeGIR-Ausbildungsstätte zu werden. Wir bereiten das gerade umfassend vor. Ich hoffe, dass die Zertifizierung 
auch bei den Patienten etwas auslöst. Insbesondere wünsche ich mir, dass die Patienten ein Bewusstsein dafür entwickeln, dass zu einer modernen Medizin auch interventionelle Therapien durch Radiologen gehören.

Wie aufwändig war die Zertifizierung für Ihr Institut - haben Sie bereits andere Zertifizierungen inne, die auf dem Weg geholfen haben?

Natürlich ist die Vorbereitung auf die Audits, die ja ein wichtiger Bestand von allen Zertifizierungen sind, sehr zeitaufwändig. Da wir aber schon seit 2008 unsere Daten im BQS (Datenbank zur Qualitätssicherungs-Dokumentation, Anm. d. Red.) systematisch erfassen, hielt sich der Aufwand in überschaubaren Grenzen. Wenn sich eine Klinik parallel als Gefäßzentrum zertifizieren lässt, liegen die meisten relevanten Zahlen bereits vor. So war es auch bei uns. Neben dem zertifizierten Gefäßzentrum haben wir auch ein zertifiziertes Brustzentrum, ein Stroke-Zentrum, ein Trauma- und Endoprothetik-Zentrum sowie ein Darmund Pankreaszentrum. Daran erkennt man, wie hochspezialisiert sich die Medizin entwickelt hat.

\section{WEITERE INFORMATIONEN}

Die DeGIR-Zertifizierung können Kliniken und radiologische Institute mit der „Zertifizierung zum Interdisziplinären Gefäßzentrum“ kombinieren und damit Kosten sparen. Diese wird durch die Deutsche Röntgengesellschaft gemeinsam mit der Deutschen Gesellschaft für Gefäßchirurgie und Gefäßmedizin (DGG) und der Deutschen Gesellschaft für Angiologie - Gesellschaft für Gefäßmedizin e. V. (DGA) angeboten.

Wie eine Zertifizierung zum „DeGIRZentrum für interventionelle Gefäßmedizin und minimal-invasive Therapie“ abläuft und welche Anforderungen Institute erfüllen müssen, erfahren Sie auf der Website der DeGIR:

www.degir.de > Zentren > DeGIR-Zentren 